\title{
Geometric least squares means ratios for the analysis of Plasmodium falciparum in vitro susceptibility to antimalarial drugs Michel Vaillant ${ }^{* \dagger 1,2}$ and Piero Olliaro ${ }^{\dagger 2,3}$
}

\author{
Address: ${ }^{1}$ Clinical Epidemiology and Public Health Unit, Centre for Health Studies, Centre de Recherche Publique (CRP)-Santé, Luxembourg, \\ ${ }^{2}$ Unité 3677, Bases thérapeutiques des inflammations et infections, Université Victor Segalen Bordeaux II, Bordeaux, France and ${ }^{3}$ UNICEF/UNDP/ \\ WB/WHO Special Programme for Research \& Training in Tropical Disease, Geneva, Switzerland \\ Email: Michel Vaillant* - michel.vaillant@crp-sante.lu; Piero Olliaro - olliarop@who.int \\ * Corresponding author †Equal contributors
}

Published: 26 November 2007

Malaria Journal 2007, 6:156 doi:10.1186/1475-2875-6-156
Received: I June 2007

Accepted: 26 November 2007

This article is available from: http://www.malariajournal.com/content/6/1/156

(c) 2007 Vaillant and Olliaro; licensee BioMed Central Ltd.

This is an Open Access article distributed under the terms of the Creative Commons Attribution License (http://creativecommons.org/licenses/by/2.0), which permits unrestricted use, distribution, and reproduction in any medium, provided the original work is properly cited.

\begin{abstract}
Background: The susceptibility of microbes such as Plasmodium falciparum to drugs is measured in vitro as the concentration of the drug achieving $50 \%$ of maximum effect $\left(\mathrm{IC}_{50}\right)$; values from a population are summarized as geometric means. For antimalarial drugs, as well as for antibiotics, assessing changes in microbe susceptibility over time under drug pressure would help inform treatment policy decisions, but no standard statistical method exists as yet.

Methods: A mixed model was generated on $\log _{\mathrm{e}}$-transformed $\mathrm{IC}_{50}$ values and calculated geometric least squares means (GLSM) with $90 \%$ confidence intervals (Cls). In order to compare $1 \mathrm{CC}_{50} \mathrm{~s}$ between years, GLSM ratios (GLSMR) with $90 \% \mathrm{Cls}$ were calculated and, when both limits of the $90 \%$ Cls were below or above $100 \%$, the difference was considered statistically significant. Results were compared to those obtained from ANOVA and a generalized linear model (GLM).

Results: GLSMRs were more conservative than ANOVA and resulted in lower levels of statistical significance. The GLSMRs approach allowed for random effect and adjustment for multiple comparisons. GLM was limited in the number of year-to-year comparisons by the need for a single reference year. The three analyses yielded generally consistent results.

Conclusion: A robust analytical method can palliate inherent limitations of in vitro sensitivity testing. The random effects GLSMRs with adjustment for multiple comparisons and $90 \% \mathrm{Cls}$ require only assumptions on the mixed model to be applied. Results are easy to display graphically and to interpret. The GLMSRs should be considered as an option for monitoring changes in drug susceptibility of $P$. falciparum malaria and other microbes.
\end{abstract}

\section{Background}

Plasmodium falciparum, the species causing most of the malaria burden in the world, can be grown in culture, which makes it possible to measure parasite drug susceptibility in vitro [1]. Results are generally expressed as $\mathrm{IC}_{50}$ (the concentration achieving 50\% of maximum effect). Turnidge et al [2] presented a new method to evaluate cut- off values to define antibacterial resistance. However, while methodologies are well established for antimicrobials [3], it is not possible, for the majority of drugs, to confidently classify a strain as resistant or sensitive because validated thresholds are not available, unlike the situation with antibiotics [4]. 
Of particular interest in $P$. falciparum drug susceptibility is not the sensitivity profile of an isolate from a given patient, but rather the monitoring of the sensitivity patterns to drugs when submitted to drug pressure in a given area [5]. This information may contribute to inform decision as to choice of drugs for the treatment of malaria. However, evaluating trends over time poses methodological problems, mainly because (i) the high variability of results makes conventional statistical methods, such as the analysis of variance (ANOVA), inadequate; (ii) the statistical unit is not the individual patient isolate but the composite population of parasites within an individual. Due to this distribution variability, these data are usually presented as geometric mean. A key issue in the analysis of these data is detecting trends over time. However, there is still no standard statistical approach for this [6], without loss of information.

Methods used in pharmacokinetic studies deal with similar problems in dealing with datasets with respect to drug disposition in an individual or a population of individuals [7-9]. Bioequivalence between drug formulations is typically assessed by using the analysis of variance of $\log _{e^{-}}$ transformed data from a cross-over design with the null hypothesis expressed by: $\mathrm{H}_{0}: \mu_{\mathrm{T}}=\mu_{\mathrm{R}}$ where $\mu_{\mathrm{T}}$ and $\mu_{\mathrm{R}}$ represent the log-transformed expected bioavailability parameters of the test and reference formulations respectively $[10,11]$. A 90\% confidence interval (CI) for the ratio test/reference for bioavailability parameters is constructed by using the equation: $\mu_{T}-\mu_{R} \pm S \sqrt{\frac{2}{n t_{0.05(1), v}}}$ where $S$ is the square root of the mean square error from the analysis of variance, $\mathrm{n}$ is the number of subjects per period, $\mathrm{t}_{0.05(1)}$ is the critical value of $\mathrm{t}$ at $\alpha=0.05$ with $v$ the degrees of freedom.

In the case of susceptibility testing conducted over time, when subjects are grouped by the year of sampling, the variance in the ANOVA of parasite susceptibility (expressed as $\mathrm{IC}_{50}$ ) can be separated out into the contributions of the parasite, the subject and the time of measurement. The ANOVA will allow for the year of measurement and test whether the variability between years occurs at random or not. The ratio of the mean sum of squares of the parameter (e.g. $\left.\mathrm{IC}_{50}\right)$ to the error mean sum of squares in the ANOVA will give an F-statistic to test the null hypothesis: $\mathrm{H}_{0}: \mu_{\text {year }}=\mu_{\text {year } 0}$. This will provide a test of whether the arithmetic mean of $\mathrm{IC}_{50} \mathrm{~s}$ measured from a given year is identical to the mean of $\mathrm{IC}_{50}$ s obtained in the reference year.
An underlying assumption in order to use the ANOVA is the normality of the residuals (the difference between an individual value and the mean of the sample it belongs to, $x_{i}-\bar{x}$.) However, verifying the null hypothesis of identity between means may not be possible with distributions of residuals that are generally neither normal nor log-normal. Furthermore, the sample size is most often too small with respect to the variance for the comparison of means. The high variability of data leads to large error variability in terms of error sum of squares. Thus, the detection of a difference will be difficult to interpret since it will be a function of the variability of data and sample size for each year. Mixed models can be used when there are different levels of clustering in the observations. One can assume that there is a grouping per year and a random part of measurements within years due to the subject and the parasites strains the subject is infected with. It allows the user to analyse samples (here: years) with unequal sample sizes and to relax the assumption of independently and identically distributed residuals while accounting for the data structure in a more flexible way [12].

For malaria, with the introduction of new treatment regimens such as the Artemisinin-containing Combination Therapies (ACTs) [13], it is important to evaluate whether, with parasites being exposed to drug pressure, the amounts of drug needed to inhibit parasite growth departs from that of a reference year, prior to and during deployment to monitor the evolution of drug susceptibility. In addition, appropriate statistical methods are needed to account for the variability in the determination of $\mathrm{IC}_{50} \mathrm{~s}$ in order to properly inform treatment policy decisions.

Several approaches were explored to describe the trends over time of parasite in vitro drug susceptibility of the parasite using a dataset collected in Casamance (south-western Senegal) during 2000-2004 slightly adapted for the purpose from Agnamey et al [14].

\section{Methods \\ Mixed model}

A fixed effect model can be expressed as $y_{i j}=\mu+t_{j}+e_{i j}$ where $\mathrm{j}$ is the year of measurement, $\mathrm{y}_{\mathrm{ij}}$ the observation on year $\mathrm{j}$ for patient $\mathrm{I}, \mu$ the overall mean (also referred to as intercept in statistical softwares), $t_{j}$ the relative effect of year $j_{,}, \mu+t_{j}$ is the mean effect for the year $j$ and $e_{i j}$ the residual variance for year $j$ on the $i^{\text {th }}$ patient $[12]$. The model allows for the patient effect, in which case the formula becomes $y_{i j}=\mu+p_{i}+t_{j}+e_{i j}$ where $p_{i}$ represents the $i^{\text {th }}$ patient effect. Instead of defining some effects as constants in the model, one could consider them as arising from independent samples with a normal distribution[12], i.e. 
as random effects. The model containing both fixed and random effects can then be referred to as a mixed model $[12,15]$. The underlying assumptions to using mixed models are: normally distributed residuals, normally distributed random effects and residuals independent of the random effects.

\section{GLSMRs calculations}

A mixed linear model of $\log _{\mathrm{e}}$-transformed values was estimated whereby the year was considered as fixed and the intercept as a random effect. The isolates were from different subjects each year.

From the mixed model, $\mathrm{t}$ statistics of standardized pair wise differences were calculated as $\bar{y}_{i}-\bar{y}_{j} / \hat{\sigma}_{i j}$ where $i$ and $j$ are the indices of two years, $\bar{y}_{i}$ and $\bar{y}_{j}$ are the least square means (LSM) for years $i$ and $j$ and $\hat{\sigma}_{i j}$ is the square-root of the estimated variance of $\bar{y}_{i}-\bar{y}_{j}$. In this model, LSMs are predicted population means from $\log _{\mathrm{e}}$-transformed values. Consequently, assuming that $\log _{e} \frac{y_{i}}{y_{j}}=\log _{e} y_{i}-\log _{e} y_{j}$ and that the geometric mean is the antilog of the mean of $\log _{\mathrm{e}}$-transformed values, the geometric least squares means ratio (GLSMR) can be calculated with the antilog of the $\bar{y}_{i}-\bar{y}_{j}$ (LSMs differences) extracted from the model (where $y_{i}$ and $y_{j}$ can be expressed as linear combination $l_{i}^{\prime} b$ and $l_{j}^{\prime} b$ of the parameter estimates). From these linear combinations the parameter estimates that define the LSMs, $\hat{\sigma}_{i j}$, (i.e. the standard deviation of the LSMs difference), can be estimated by $\hat{\sigma}_{i j}^{2}=s^{2} l_{i}^{\prime}\left(X^{\prime} X\right)^{-} l_{j}$. The confidence interval can be derived as $\left(\bar{y}_{i}-\bar{y}_{j}\right) \pm t_{v, \frac{\alpha}{2}} \sigma_{i j}$.

GLSM ratios (GLSMRs) were calculated for each betweenyear comparison. An adjustment for multiple comparisons was done in order to control for the overall type 1 error rate using the Tukey-Kramer method (chosen because it allows for unequal sample size between years). GLSMRs were considered statistically different if both bounds of the CIs fell on either side of the value of 1 (or $100 \%$ in percentage values). Previously [14], we had used GLSMRs calculated without this adjustment and evaluated the $95 \%$ CIs.

\section{Standard statistical methods}

Standard methods such as the one-way ANOVA were also used with the year as fixed factor to analyse the variations of $\mathrm{IC}_{50} \mathrm{~s}$ over time. For non-normally distributed data (significant Kolmogorov-Smirnov test), a log $e^{-t r a n s f o r m a t i o n ~}$ was applied. The Levene test for homogeneity of the variance was used and, in case of non-homogeneity, a Welch adjustment for the ANOVA. A non-parametric KruskallWallis sign rank test was used when parametric analyses were not suitable. Pair-wise mean comparisons between years for each treatment were carried out following ANOVA with a Tukey adjustment. Normality of residuals was checked with a non significant Shapiro-Wilk test and normal probability plots. Concurrently a generalized linear model (GLM) was also estimated with the year as fixed factor using a normal probability function and an identity link function parameterization [16].

A p-value of $<0.05$ was considered statistically significant. All tests were two-tailed. Statistical analyses were carried out with the statistical package SAS ${ }^{\circledast}$ System version 9.1.3 (SAS Institute, Cary, NC, USA).

\section{Dataset}

The dataset is an update of the one described in Agnamey et al [14]. Briefly, in vitro susceptibility of local isolates to chloroquine (CQ), quinine (QN), artemisinin (ART) and the amodiaquine metabolite monodesethylamodiaquine (MdAQ) were monitored the using the DELI test [17] before (1997) and during the deployment (2000-2004) of artesunate+amodiaquine combination in Mlomp, a village in the district of Oussouye in Casamance, Southern Senegal, where malaria is mesoendemic (25 infective bites/person-year) and transmission occurs year-round with a peak during the rainy season (July-December). Samples for the in vitro assay were from consecutive patients recruited as part of an observational study [18] with a $P$. falciparum mono-infection and parasitaemia $\geq$ $0.2 \%[19]$.

\section{Results}

$\mathrm{IC}_{50} \mathrm{~s}$ from 242 subjects for CQ, 250 subjects for QN, 236 subjects for MdAQ and 183 subjects for ART were used in these statistical analyses [14]. The number of subjects with in vitro results was different among years and products tested (Table 1). Means with two standard deviations along with data distributions are plotted in Figure 1. Mean, Geometric means and GLSMs are presented together in Table 1. For all products except log-transformed ART, values were not normally distributed (Kolmogorov-Smirnov test $\mathrm{p}<0.05)$.

\section{Results of the analyses using the different methods}

The ANOVA pair wise means comparisons (Table 2) showed no significant differences for $\mathrm{CQ} \mathrm{IC}_{50}$ values. For MdAQ, statistically significant increases were observed between 1997 and 2000-2004, while there were decreases in $\mathrm{IC}_{50} \mathrm{~s}$ between 2000-2004, 2001-2004 and 
Table I: Means of raw and $\log _{\mathrm{e}}$-transformed $I C_{50} s$, geometric means and Geometric Least Squares Means.

\begin{tabular}{|c|c|c|c|c|c|c|c|c|c|c|c|c|c|c|c|c|c|c|c|}
\hline \multicolumn{4}{|c|}{1997} & \multicolumn{3}{|c|}{2000} & \multicolumn{3}{|c|}{2001} & \multicolumn{3}{|c|}{2002} & \multicolumn{3}{|c|}{2003} & \multicolumn{3}{|c|}{2004} & \multirow{2}{*}{$\begin{array}{c}\text { Normality } \\
\text { p-value }\end{array}$} \\
\hline Variable & $\mathrm{N}$ & Mean & STD & $\mathrm{N}$ & Mean & STD & $\mathrm{N}$ & Mean & STD & $N$ & Mean & STD & $\mathrm{N}$ & Mean & STD & $N$ & Mean & STD & \\
\hline \multicolumn{20}{|c|}{ Means (raw values) } \\
\hline $\mathrm{CQ}$ & 31 & 236 & 203.4 & 33 & 173.2 & 223 & 31 & 251.4 & 236.7 & 46 & 131.3 & 104 & 34 & 143.8 & 91.03 & 67 & 171.5 & 157.2 & 0.01 \\
\hline $\mathrm{QN}$ & 46 & 234.6 & 150.2 & 28 & 305.8 & 210.4 & 31 & 444 & 286.2 & 40 & 252.6 & 273.6 & 34 & 280.8 & 162.8 & 58 & 207.5 & 149.8 & 0.01 \\
\hline MdAQ & 45 & 12.8 & 12.4 & 33 & 85.1 & 167.8 & 31 & 57.7 & 63.2 & 43 & 42.9 & 32.3 & 34 & 31.1 & 21.3 & 64 & 21.7 & 17.6 & 0.01 \\
\hline ART & 10 & 3.6 & 0.8 & 31 & 9.8 & 12.0 & 31 & 6.1 & 6.6 & 40 & 5.7 & 4.5 & 34 & 7.4 & 5.9 & 36 & 7.5 & 5.0 & 0.01 \\
\hline \multicolumn{20}{|c|}{ Means (natural log values) } \\
\hline $\mathrm{CQ}$ & 31 & 4.9 & 1.4 & 33 & 4.4 & 1.2 & 31 & 5 & 1.2 & 46 & 4.5 & 0.9 & 34 & 4.7 & 0.8 & 67 & 4.7 & 1 & 0.01 \\
\hline $\mathrm{QN}$ & 46 & 5.2 & 0.8 & 28 & 5.5 & 0.8 & 31 & 5.9 & 0.7 & 40 & 5.1 & 0.9 & 34 & 5.5 & 0.5 & 58 & 5.1 & 0.8 & 0.04 \\
\hline MdAQ & 45 & 2.1 & 0.9 & 33 & 3.8 & 1.0 & 31 & 3.6 & 0.9 & 43 & 3.5 & 0.7 & 34 & 3.2 & 0.6 & 64 & 2.8 & 0.9 & 0.04 \\
\hline ART & 10 & 1.2 & 0.3 & 31 & 1.8 & 1.0 & 31 & 1.3 & 1 & 40 & 1.3 & 1.0 & 34 & 1.8 & 0.6 & 36 & 1.8 & 0.7 & 0.15 \\
\hline \multicolumn{20}{|c|}{ Geometric means } \\
\hline $\mathrm{CQ}$ & 31 & 130.3 & 3.9 & 33 & 82.3 & 3.5 & 31 & 148.4 & 3.2 & 46 & 93.7 & 2.5 & 34 & 112.2 & 2.2 & 67 & 115.6 & 2.7 & - \\
\hline $\mathrm{QN}$ & 46 & 181.3 & 2.3 & 28 & 237.5 & 2.2 & 31 & 350.7 & 2.1 & 40 & 169.0 & 2.6 & 34 & 244.7 & 1.7 & 58 & 162.4 & 2.1 & - \\
\hline MdAQ & 45 & 8.5 & 2.5 & 33 & 43.8 & 2.7 & 31 & 38.5 & 2.4 & 43 & 33.1 & 2.1 & 34 & 25.3 & 1.9 & 64 & 15.8 & 2.4 & - \\
\hline ART & 10 & 3.5 & 1.3 & 31 & 5.8 & 2.7 & 31 & 3.9 & 2.7 & 40 & 3.9 & 2.8 & 34 & 6.1 & 1.9 & 36 & 6.0 & 2.0 & - \\
\hline \multicolumn{20}{|c|}{ Geometric Least Squares Means } \\
\hline $\mathrm{CQ}$ & 31 & 130.8 & 1.2 & 33 & 82.6 & 1.2 & 31 & 148.4 & 1.2 & 46 & 93.3 & 1.2 & 34 & 112.7 & 1.2 & 67 & 115 & 1.1 & - \\
\hline QN & 46 & 181.3 & 1.1 & 28 & 238.2 & 1.2 & 31 & 350.1 & 1.1 & 40 & 168.4 & I.I & 34 & 245 & 1.1 & 58 & 162.2 & 1.1 & - \\
\hline MdAQ & 45 & 8.5 & 1.1 & 33 & 44 & 1.2 & 31 & 38.5 & 1.2 & 43 & 33.1 & 1.1 & 34 & 25.3 & 1.2 & 64 & 15.9 & 1.1 & - \\
\hline ART & 10 & 3.5 & 1.3 & 31 & 5.8 & 1.2 & 31 & 3.9 & 1.2 & 40 & 3.9 & I.I & 34 & 6.1 & 1.2 & 36 & 6.0 & 1.1 & - \\
\hline
\end{tabular}

$\mathrm{P}$-values are from the Kolmogorov-Smirnov test for normality. $\mathrm{CQ}=$ chloroquine; $\mathrm{QN}=$ quinine; $\mathrm{MdAQ}=$ monodesethylamodiaquine; $\mathrm{ART}=$ artemisinin

2002-2004. For QN, a statistically significant increase was observed between 1997 and 2001, and a decrease between 2001-2002 and 2001-2004 (Figure 1). For ART, no statistically significant differences were observed.

Using the GLM with 1997 as the reference year (Table 3), no relationship was found for CQ and ART, a significant positive estimate for MdAQ $\left(\mathrm{IC}_{50} \mathrm{~s}\right.$ decreased $)$ and a significant positive estimate for QN in 2001, followed by a negative estimate ( $\mathrm{IC}_{50} \mathrm{~s}$ increased) in 2002.

GLSMR (Table 4) of CQ $\mathrm{IC}_{50}$ s showed no significant differences, consistent with a stable response to CQ over the study period (Figure 2). For MdAQ, there were statistically significant increases between 1997 and 2000-2004. From 2000 to 2004, there was a decrease in $\mathrm{IC}_{50} \mathrm{~s}$ which was significant between 2000 and 2004, 2001 and 2004, as well as 2002 and 2004. QN showed an increase between 1997 and 2001 and 2001-2002, and a significant decrease between 2002-2004. For ART, no statistically significant changes were found (Figure 2).

\section{Comparison of the results obtained with the different methods used}

GLSMRs and ANOVA generated consistent results. With the GLM, results were similar to the two other tests for CQ and ART; for MdAQ, the GLM identified only a decrease between 1997 and 2001; for QN, it showed an increase between 1997 and 2001, while the ANOVA and GLSMRs revealed a decrease for 2001-2002 and 2001-2004 periods respectively.

For comparison, when this set of data is analysed with GLSMRs with no random intercept and 95\%CIs calculated without adjustment for multiple comparisons, results are slightly different from the current GLSMRs. With the former, significant differences are found for 1997-2000 for CQ and ART, between 2001-2002 for CQ and 2002-2003 for ART. There are two significant differences with 2000 as reference year in comparison with 2002 and 2004, which are not found with the current GLSMRs. Similarly, 2001 is different from 2003 and 2003 from 2004. In the case of ART, the year 2003 is significantly different from the others while it was not with the current GLSMRs

\section{Discussion}

In this study, three different statistical methods to assess changes of $\mathrm{IC}_{50}$ over time (ANOVA, GLM, GLSMRs) were compared. The use of data from a single site of moderate to high transmission (25 infecting bites per person-year), and with consistent treatment policies and practices, meant that all patients were expected to be infected with parasites having been under the same degree of drug pressure. 

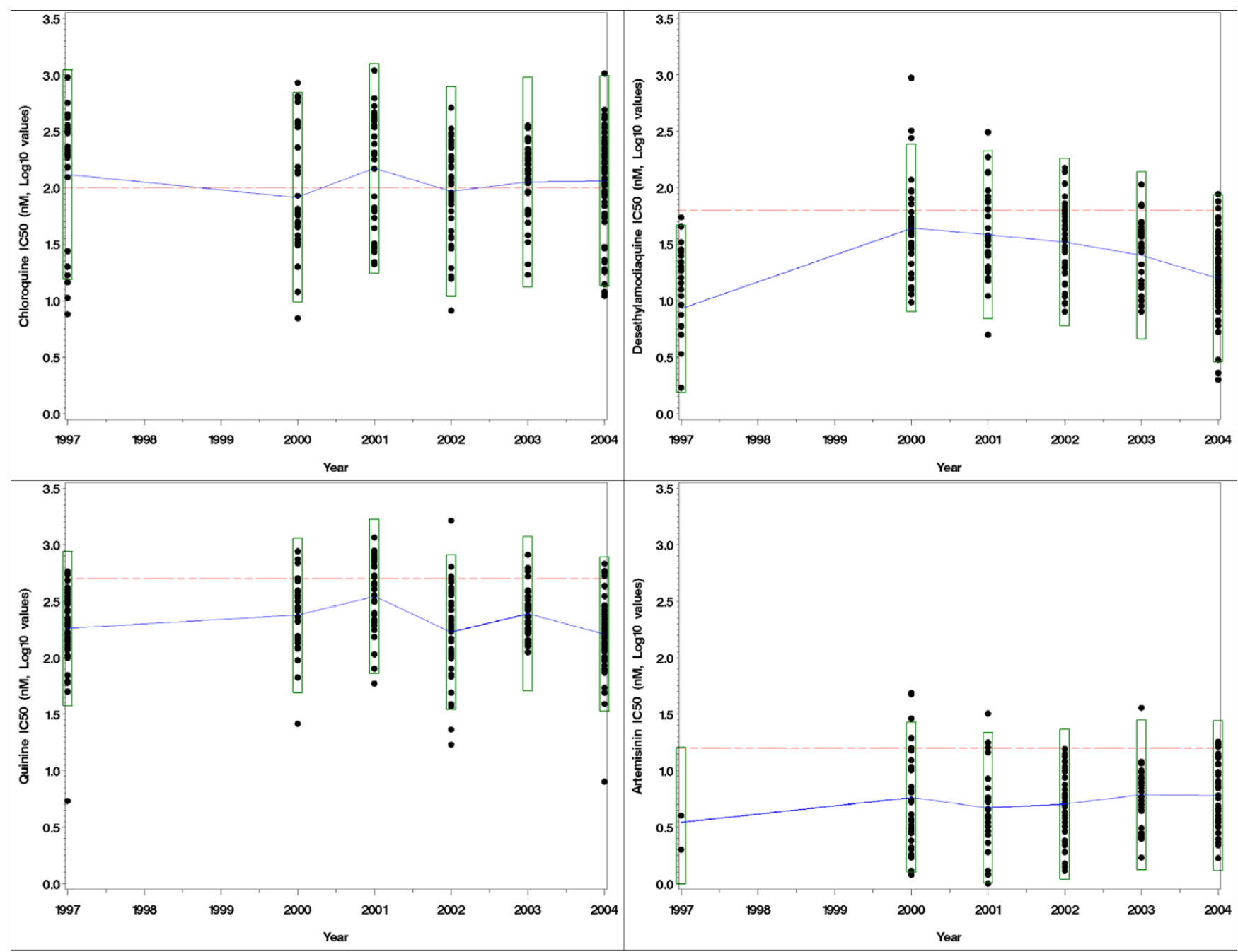

\section{Figure I}

Scatterplots of the $\log _{e}$-transformed values of the quinine (QN), chloroquine (CQ), monodesethylamodiaquine (MdAQ), and artemisinin (ART) $I C_{50}$ s between years. The straight line is the cut-off value for susceptibility to the products (here: $100 \mathrm{nM}$ for $\mathrm{CQ}, 60 \mathrm{nM}$ for AQ, $500 \mathrm{nM}$ for QN and $15 \mathrm{nM}$ for ART [4]). The means of each year are connected with a trend line. The box around each year distribution of values represent two standard deviation of the mean.

\section{Normality assumption}

Data were not normally distributed, even after $\log _{\mathrm{e}}$-transformation, for all drugs except $\log _{\mathrm{e}}$-transformed ART. The ANOVA can be used on non-normally distributed values (although in this case conclusions are less robust), and allows for multiple pair wise comparisons adjustment (but then residuals must be checked for independence and normality as well as homosedasticity, i.e the condition whereby variances are equal.) The GLM requires normally distributed values when using an identity link function (i.e. an assumption of normal distribution for the studied parameter) for comparison with a unique reference (baseline) value. For non-normally distributed data, a different model must be used for each different ref- erence value of the independent factor. The GLSMRs approach can use non normally distributed values and allows adjusted multiple comparisons between years.

\section{GLSMR}

The GLSMR had broader applicability than the other methods because, even if a mixed model is used to obtain the LSMs, it does not require either normally distributed $\mathrm{IC}_{50} \mathrm{~s}$ or variance homogeneity. However, one needs to verify the assumptions needed for a mixed model such as the normality of the residuals, the normality of the random effects, and the independence of the residuals and the random effects. As $\log _{\mathrm{e}}$-transformation serves the purpose of deriving GLSMs of the results, the linear mixed 
Table 2: Pairwise Least Squares Means comparisons following ANOVA of chloroquine (CQ), monodesethylamodiaquine (MdAQ), Quinine (QN) and Artemisinin (ART) log ${ }^{-}$ transformed $\mathrm{IC}_{50}(\mathrm{nM})$ between years.

\begin{tabular}{|c|c|c|c|c|c|c|c|c|c|c|c|c|c|c|c|c|}
\hline & & MdAQ & & & & $\mathrm{CQ}$ & & & & QN & & & & ART & & \\
\hline year Comparison & $\begin{array}{c}\text { Difference } \\
\text { Between Means }\end{array}$ & $\begin{array}{l}\text { Simultaneous } 95 \% \\
\text { Confidence Limits }\end{array}$ & & Sig. & $\begin{array}{c}\text { Difference } \\
\text { Between Means }\end{array}$ & $\begin{array}{l}\text { Simultaneous } 95 \% \\
\text { Confidence Limits }\end{array}$ & & Sig. & $\begin{array}{c}\text { Difference } \\
\text { Between Means }\end{array}$ & $\begin{array}{l}\text { Simultaneous } 95 \% \\
\text { Confidence Limits }\end{array}$ & & Sig. & $\begin{array}{c}\text { Difference } \\
\text { Between Means }\end{array}$ & $\begin{array}{l}\text { Simultaneous } 95 \% \\
\text { Confidence Limits }\end{array}$ & & Sig. \\
\hline $1997-2000$ & -1.6 & -2.3 & -1.0 & $* * *$ & 0.5 & -0.4 & 1.4 & & -0.3 & -0.9 & 0.4 & & -0.5 & -1.6 & 0.5 & \\
\hline $1997-2001$ & -1.5 & -2.2 & -0.8 & *** & -0.1 & -1.0 & 0.8 & & -0.7 & -1.3 & 0.0 & 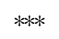 & -0.1 & -1.2 & 0.9 & \\
\hline $1997-2002$ & -1.4 & -2.0 & -0.7 & $* * *$ & 0.3 & -0.5 & 1.2 & & 0.1 & -0.5 & 0.6 & & -0.1 & -1.1 & 0.9 & \\
\hline $1997-2003$ & -1.1 & -1.7 & -0.4 & $* * *$ & 0.1 & -0.7 & 1.0 & & -0.3 & -0.9 & 0.3 & & -0.6 & -1.6 & 0.5 & \\
\hline $1997-2004$ & -0.6 & -1.2 & -0.1 & $* * *$ & 0.1 & -0.6 & 0.9 & & 0.1 & -0.4 & 0.6 & & -0.5 & -1.6 & 0.5 & \\
\hline $2000-1997$ & 1.6 & 1.0 & 2.3 & $* * *$ & -0.5 & -1.4 & 0.4 & & 0.3 & -0.4 & 0.9 & & 0.5 & -0.5 & 1.6 & \\
\hline $2000-2001$ & 0.1 & -0.6 & 0.8 & & -0.6 & -1.5 & 0.3 & & -0.4 & -1.1 & 0.3 & & 0.4 & -0.3 & 1.1 & \\
\hline $2000-2002$ & 0.3 & -0.4 & 0.9 & & -0.1 & -0.9 & 0.7 & & 0.3 & -0.3 & 1.0 & & 0.4 & -0.3 & 1.1 & \\
\hline $2000-2003$ & 0.6 & -0.1 & 1.2 & & -0.3 & -1.2 & 0.6 & & 0.0 & -0.7 & 0.6 & & -0.1 & -0.8 & 0.7 & \\
\hline $2000-2004$ & 1.0 & 0.4 & 1.6 & $* * *$ & -0.3 & -1.1 & 0.4 & & 0.4 & -0.2 & 1.0 & & 0.0 & -0.7 & 0.7 & \\
\hline $2001-1997$ & 1.5 & 0.8 & 2.2 & $* * *$ & 0.1 & -0.8 & 1.0 & & 0.7 & 0.0 & 1.3 & 桃 & 0.1 & -0.9 & 1.2 & \\
\hline $2001-2000$ & -0.1 & -0.8 & 0.6 & & 0.6 & -0.3 & 1.5 & & 0.4 & -0.3 & 1.1 & & -0.4 & -1.1 & 0.3 & \\
\hline $2001-2002$ & 0.2 & -0.5 & 0.8 & & 0.5 & -0.4 & 1.3 & & 0.7 & 0.1 & 1.4 & $* * *$ & 0.0 & -0.7 & 0.7 & \\
\hline $2001-2003$ & 0.4 & -0.3 & 1.1 & & 0.3 & -0.6 & 1.2 & & 0.4 & -0.3 & 1.0 & & -0.5 & -1.2 & 0.3 & \\
\hline $2001-2004$ & 0.9 & 0.3 & 1.5 & $* * *$ & 0.3 & -0.5 & 1.0 & & 0.8 & 0.2 & 1.4 & 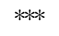 & -0.4 & -1.2 & 0.3 & \\
\hline $2002-1997$ & 1.4 & 0.7 & 2.0 & $* * *$ & -0.3 & -1.2 & 0.5 & & -0.1 & -0.6 & 0.5 & & 0.1 & -0.9 & 1.1 & \\
\hline $2002-2000$ & -0.3 & -0.9 & 0.4 & & 0.1 & -0.7 & 0.9 & & -0.3 & -1.0 & 0.3 & & -0.4 & -1.1 & 0.3 & \\
\hline $2002-2001$ & -0.2 & -0.8 & 0.5 & & -0.5 & -1.3 & 0.4 & & -0.7 & -1.4 & -0.1 & **** & 0.0 & -0.7 & 0.7 & \\
\hline $2002-2003$ & 0.3 & -0.4 & 0.9 & & -0.2 & -1.0 & 0.6 & & -0.4 & -1.0 & 0.2 & & -0.5 & -1.1 & 0.2 & \\
\hline $2002-2004$ & 0.7 & 0.2 & 1.3 & $* * *$ & -0.2 & -0.9 & 0.5 & & 0.0 & -0.5 & 0.6 & & -0.4 & -1.1 & 0.2 & \\
\hline $2003-1997$ & 1.1 & 0.4 & 1.7 & $* * *$ & -0.1 & -1.0 & 0.7 & & 0.3 & -0.3 & 0.9 & & 0.6 & -0.5 & 1.6 & \\
\hline $2003-2000$ & -0.6 & -1.2 & 0.1 & & 0.3 & -0.6 & 1.2 & & 0.0 & -0.6 & 0.7 & & 0.1 & -0.7 & 0.8 & \\
\hline $2003-2001$ & -0.4 & -1.1 & 0.3 & & -0.3 & -1.2 & 0.6 & & -0.4 & -1.0 & 0.3 & & 0.5 & -0.3 & 1.2 & \\
\hline $2003-2002$ & -0.3 & -0.9 & 0.4 & & 0.2 & -0.6 & 1.0 & & 0.4 & -0.2 & 1.0 & & 0.5 & -0.2 & 1.1 & \\
\hline $2003-2004$ & 0.5 & -0.1 & 1.1 & & 0.0 & -0.8 & 0.7 & & 0.4 & -0.2 & 1.0 & & 0.0 & -0.7 & 0.7 & \\
\hline $2004-1997$ & 0.6 & 0.1 & 1.2 & $* * *$ & -0.1 & -0.9 & 0.6 & & -0.1 & -0.6 & 0.4 & & 0.5 & -0.5 & 1.6 & \\
\hline $2004-2000$ & -1.0 & -1.6 & -0.4 & $* * *$ & 0.3 & -0.4 & 1.1 & & -0.4 & -1.0 & 0.2 & & 0.0 & -0.7 & 0.7 & \\
\hline $2004-2001$ & -0.9 & -1.5 & -0.3 & $* * *$ & -0.3 & -1.0 & 0.5 & & -0.8 & -1.4 & -0.2 & $* * *$ & 0.4 & -0.3 & 1.2 & \\
\hline $2004-2002$ & -0.7 & -1.3 & -0.2 & 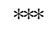 & 0.2 & -0.5 & 0.9 & & 0.0 & -0.6 & 0.5 & & 0.4 & -0.2 & 1.1 & \\
\hline $2004-2003$ & -0.5 & -1.1 & 0.1 & & 0.0 & -0.7 & 0.8 & & -0.4 & -1.0 & 0.2 & & 0.0 & -0.7 & 0.7 & \\
\hline
\end{tabular}


Table 3: Generalized linear model (SAS System Proc Genmod) of chloroquine (CQ), monodesethylamodiaquine (MdAQ), quinine $(\mathrm{QN})$ and artemisinin (ART) IC 50 (nM) with the year as independent parameter (log $_{\mathrm{e}}$-transformed values and 1997 as the reference value).

\begin{tabular}{|c|c|c|c|c|c|c|c|c|c|c|c|c|c|}
\hline \multirow[b]{2}{*}{ Parameter } & & \multicolumn{3}{|c|}{$\mathrm{CQ}$} & \multicolumn{3}{|c|}{ MdAQ } & \multicolumn{3}{|c|}{$\mathrm{QN}$} & \multicolumn{3}{|c|}{ ART } \\
\hline & & Estimate & Std Error & $\mathrm{P}$ & Estimate & Std Error & $\mathrm{P}$ & Estimate & Std Error & $\mathrm{P}$ & Estimate & Std Error & $P$ \\
\hline Intercept & & 4.9 & 0.2 & $<.0001$ & 2.1 & 0.1 & $<.0001$ & 5.2 & 0.1 & $<.0001$ & 1.2 & 0.3 & $<.0001$ \\
\hline \multirow{5}{*}{ year* } & 2000 & -0.5 & 0.3 & N.S. & 1.6 & 0.2 & $<.0001$ & 0.3 & 0.2 & N.S. & 0.5 & 0.3 & N.S. \\
\hline & 2001 & 0.1 & 0.3 & N.S. & 1.5 & 0.2 & $<.0001$ & 0.7 & 0.2 & 0.0003 & 0.1 & 0.3 & N.S. \\
\hline & 2002 & -0.3 & 0.2 & N.S. & 1.4 & 0.2 & $<.0001$ & -0.1 & 0.2 & N.S. & 0.1 & 0.3 & N.S. \\
\hline & 2003 & -0.1 & 0.3 & N.S. & I.I & 0.2 & $<.0001$ & 0.3 & 0.2 & N.S. & 0.6 & 0.3 & N.S. \\
\hline & 2004 & -0.1 & 0.2 & N.S. & 0.6 & 0.2 & 0.0001 & -0.1 & 0.2 & N.S. & 0.5 & 0.3 & N.S. \\
\hline Scale & & 1.1 & 0.0 & - & 0.8 & 0.0 & - & 0.8 & 0.0 & - & 0.8 & 0.0 & - \\
\hline
\end{tabular}

model allowed to relax the assumption of independence of the model residuals and to account for the inherent variability of the data structure in a more flexible way. The in vitro test reflects the susceptibility of the whole parasite population in one isolate, and thus cannot separate the effects of the various parasite clones in a given infected subject. Therefore one cannot parameterize the withinsubject effect or within-parasite population effect in the linear mixed model. However, it can be argued that these effects are taken into account in the overall mean, which was defined as random in the model.

GLSMRs calculated at a 5\% level without an adjustment for multiple comparisons are more likely to wrongly detect a significant difference because of the multiplicity of the statistical tests performed than the GLSMRs at a $1 \%$ level and with an adjustment for multiplicity.

\section{Linear models}

ANOVA and pair wise means comparisons between years were good indicators of a difference between years. Noteworthy, in bioequivalence studies GLSMR are generally computed using an ANOVA model, but effects specified as random in a linear model are treated as a fixed factor as they serve the sole purpose of producing the corresponding expected mean squares [20]. These expected mean squares lead to the traditional ANOVA components without accounting for the random effect in the variance.

In the GLSMRs calculations, the mixed linear model was computed using restricted maximum likelihood to evaluate variance parameters, which are in general preferred to ANOVA estimates [20]. Furthermore, mixed models are commonly used when there are different levels of clustering in the observations. The sole level of grouping was the year (treated as fixed in the model), so no other particular variable (or grouping level) was defined as random. The reasons for treating the time variable as discrete (i.e. by calendar year and not as a continuous variable) are: (i) there was a 3-year gap between 1997 (the baseline) and
2000 (the first of a series of five consecutive years.); (ii) only qualitative or discrete variables allowed for the model to simply extract estimates for the different categories of the year effect; (iii) the majority of malaria cases and treatments cluster between July-November during the wet season. Hence, subjects were grouped by year and it was assumed that there was a random part of measurements within years due to the contribution of the subject and the parasite strains subjects were infected with. Specifically, the number of isolates for each year was not the same and $\mathrm{IC}_{50} \mathrm{~s}$ varied considerably from year to year (Table 1).

The GLM appears not to be suitable to compare $\mathrm{IC}_{50} \mathrm{~s}$ because it treated values as if there were repeat measures from the same subjects, while isolates came from different individuals. In addition, $\mathrm{IC}_{50} \mathrm{~s}$ were non-normally distributed despite $\log _{\mathrm{e}}$-transformation.

\section{Estimates of the IC50}

It is clear that customary approaches are not satisfactory as the difficulty in the analyses is that the data are not time series or longitudinal data. They are also not normally distributed - a necessary condition to use parametric statistical tests, and a critical point of this work. In a recently published paper, Kaddouri et al [21] developed a new inhibitory sigmoid Emax statistical model to estimate more precisely the $\mathrm{IC}_{50}$ of a given subject. However, it requires cut-off values for resistance of the studied parasites strains to a range of treatment, an element which is not easy to derive for antimalarial drugs. A Bayesian approach was also proposed recently to provide a correction of the estimate of the true $\mathrm{IC}_{50}$ [22]. This work was based on the assumption that resistance is systematically overestimated because: (i) the precision of the estimated $\mathrm{IC}_{50}$ value of the most resistant isolate will usually be the poorest of all the isolates assayed, (ii) sigmoid curve fitting or probit analysis of a unique isolate takes no account of other isolates in the series tested. This approach requires the distribution of measured $\mathrm{IC}_{50}$ to be estab- 


\begin{tabular}{|c|c|c|c|c|c|c|c|c|c|c|c|c|c|}
\hline & & & MdAQ & & & $\mathrm{CQ}$ & & & QN & & & ART & \\
\hline Variable & $\begin{array}{l}2 \text { one-sided } \\
90 \% \text { limits }\end{array}$ & $\begin{array}{l}\text { GLS Mean } \\
\text { Ratio (\%) }\end{array}$ & $90 \% \mathrm{Cl}$ & 2sided t $\mathrm{p}$-value & $\begin{array}{l}\text { GLS Mean } \\
\text { Ratio (\%) }\end{array}$ & $90 \% \mathrm{Cl}$ & 2sided t p-value & $\begin{array}{l}\text { GLS Mean } \\
\text { Ratio (\%) }\end{array}$ & $90 \% \mathrm{Cl}$ & $\begin{array}{l}2 \text { sided } \\
\mathrm{t} \text {-value }\end{array}$ & $\begin{array}{l}\text { GLS Mean } \\
\text { Ratio (\%) }\end{array}$ & $90 \% \mathrm{Cl}$ & 2sided $\mathrm{t} \mathrm{p}$-value \\
\hline 1997 vs 2000 & {$[80,125]$} & 516.7 & {$[266.4,1002.3]$} & $<0.0001$ & 63.2 & {$[25.5,156.7]$} & N.S. & 131.4 & {$[69.3,249.2]$} & N.S. & 166.8 & {$[57.2,486.0]$} & N.S. \\
\hline 1997 vs $200 \mid$ & {$[80,125]$} & 452.3 & {$[230.3,888.2]$} & $<0.0001$ & 113.5 & {$[45.1,285.4]$} & N.S. & 193.1 & [103.8.359.2] & 0.005 & 110.9 & {$[38.0,323.0]$} & N.S. \\
\hline 1997 vs 2002 & {$[80,125]$} & 388.2 & [209.6,7|9.2] & $<0.0001$ & 71.3 & {$[30.7,165.8]$} & N.S. & 92.9 & {$[52.1,165.4]$} & N.S. & 110.8 & {$[39.2,3 \mid 3.4]$} & N.S. \\
\hline 1997 vs 2003 & {$[80,125]$} & 297.1 & {$[154.0,573.0]$} & $<0.0001$ & 86.2 & {$[35.0,2 \mid 2.3]$} & N.S. & 135.1 & {$[73.9,247.2]$} & N.S. & 175.6 & {$[61.0,505.7]$} & N.S. \\
\hline 1997 vs 2004 & {$[80,125]$} & 186.3 & {$[106.1,326.9]$} & 0.003 & 88.0 & {$[40.0,193.5]$} & N.S. & 89.5 & {$[52.8,151.6]$} & N.S. & 172.5 & {$[60.3,493.5]$} & N.S. \\
\hline 2000 vs 2001 & {$[80,125]$} & 87.5 & {$[42.5,180.4]$} & N.S. & 179.6 & {$[72.4,445.3]$} & N.S. & 147.0 & {$[73.3,294.9]$} & N.S. & 66.5 & {$[31.5, \mid 40.3]$} & N.S. \\
\hline 2000 vs 2002 & {$[80,125]$} & 75.1 & {$[38.5,146.7]$} & N.S. & 112.9 & {$[49.3,258.3]$} & N.S. & 70.7 & {$[36.6,136.5]$} & N.S. & 66.4 & {$[32.9,134.2]$} & N.S. \\
\hline 2000 vs 2003 & {$[80,125]$} & 57.5 & {$[28.4,116.5]$} & N.S. & 136.4 & {$[56.1,331.1]$} & N.S. & 102.9 & {$[52.0,203.3]$} & N.S. & 105.3 & {$[50.7,218.5]$} & N.S. \\
\hline 2000 vs 2004 & {$[80,125]$} & 36.1 & {$[19.4,67.0]$} & $<0.0001$ & 139.2 & {$[64.3,301.2]$} & N.S. & 68.1 & {$[36.8,125.9]$} & N.S. & 103.4 & {$[50.3,212.5]$} & N.S. \\
\hline 2001 vs 2002 & {$[80,125]$} & 85.8 & {$[43.4,169.6]$} & N.S. & 62.8 & {$[27.0,146.1]$} & N.S. & 48.1 & {$[25.4,91.1]$} & 0.002 & 100.0 & {$[49.4,202.0]$} & N.S. \\
\hline 200 I vs 2003 & {$[80,125]$} & 65.7 & {$[32.0,134.7]$} & N.S. & 75.9 & {$[30.8,187.0]$} & N.S. & 70.0 & {$[36.0,135.8]$} & N.S. & 158.4 & {$[76.3,328.7]$} & N.S. \\
\hline 200 I vs 2004 & {$[80,125]$} & 41.2 & {$[21.9,77.5]$} & 0.000 & 77.5 & {$[35.2,170.5]$} & N.S. & 46.3 & {$[25.6,83.9]$} & $<0.0001$ & 155.6 & {$[75.7,3 \mid 9.8]$} & N.S. \\
\hline 2002 vs 2003 & {$[80,125]$} & 76.5 & {$[39.4,148.6]$} & N.S. & 120.8 & {$[53.2,274.6]$} & N.S. & 145.5 & {$[78.0,271.2]$} & N.S. & 158.5 & {$[79.8,3 \mid 4.7]$} & N.S. \\
\hline 2002 vs 2004 & {$[80,125]$} & 48.0 & {$[27.1,84.8]$} & $<0.0001$ & 123.3 & {$[61.5,247.2]$} & N.S. & 96.3 & {$[55.7,166.8]$} & N.S. & 155.7 & {$[79.2,305.9]$} & N.S. \\
\hline 2003 vs 2004 & {$[80,125]$} & 62.7 & {$[33.9,115.8]$} & N.S. & 102.1 & {$[47.5,219.2]$} & N.S. & 66.2 & {$[37.2,117.9]$} & N.S. & 98.2 & {$[48.6,198.4]$} & N.S. \\
\hline
\end{tabular}



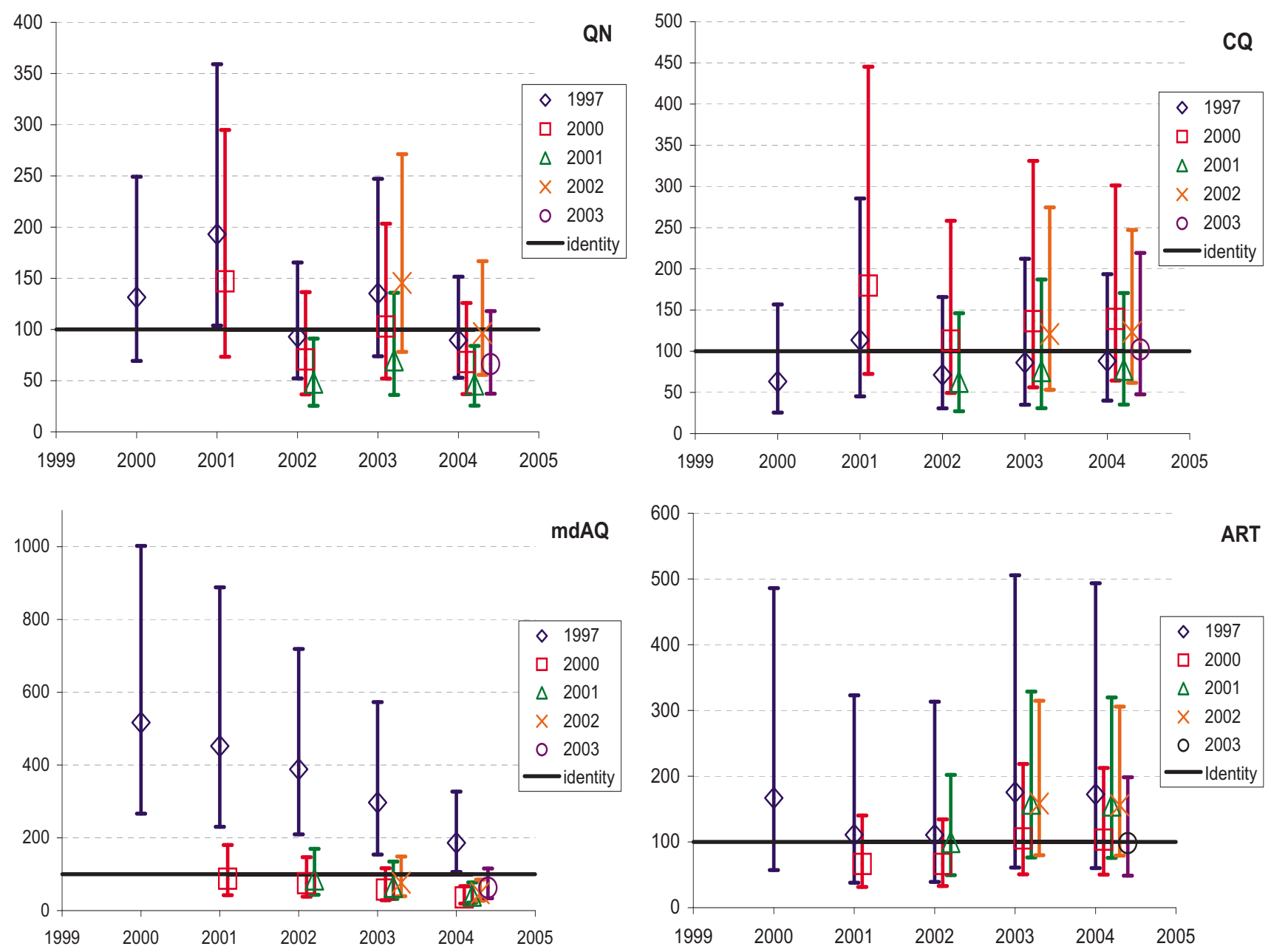

Figure 2

Geometric Least Squares Means Ratios of the $\mathrm{IC}_{50}$ s to quinine (QN), chloroquine (CQ), monodesethylamodiaquine (MdAQ), and artemisinin (ART). The legend indicates the reference year for the ratio. The $x$-axis indicates the year tested in the ratio. $A$ logarithmic scale was applied for QN.

lished before using it to produce a large number of points and estimate the median as the true $\mathrm{IC}_{50}$. In doing so, one is inevitably faced with the problem of normality and the choice to apply data transformations such as log or BoxCox [23]. This work offers an alternative way to deal with data transformation and normality condition in the context of the evolution of microbial susceptibility to drugs.

\section{Expression of results}

GLSMRs were found to be more intuitive, as results are expressed as percentage difference (increase or decrease) between two years, while with LSMs comparisons increases and decreases from the reference appear as inverted (they are marked with a negative and a positive sign, respectively). As geometric means are generally used to express $\mathrm{IC}_{50} \mathrm{~s}$ of a pool of isolates, GLSMR are naturally easier to understand and interpret than the other statistical methods tested here.

Two different plots were also produced in order to illustrate the difficulties in interpreting trends over time in drug susceptibility. Figure 1 is a traditional way of plotting the distribution of $\mathrm{IC}_{50} \mathrm{~s}$ with cut-off values above which a strain is resistant to a given drug. This makes the reader falsely interpret means against the cut-off, while there is a great variability in the data and no validated thresholds for the majority of antimalarial drugs. In addition, this display does not provide any indication of the significance (or lack thereof) of change between years. Figure 2 is based on the GLSMR results and provides a direct com- 
parison of GLSM between years; it does not need the cutoff values; it depicts visually a statistical test as the confidence intervals of each GLSMR against the line of identity between sets of data (here: years).

\section{Conclusion}

Treatment policy decisions would benefit from reliable information on changes in susceptibility of parasite or bacterial isolates to drugs over time. This entails an adequate statistical method, which can also account for the inherent variability of in vitro drug susceptibility tests (Figure 2). This is particularly important for antimalarial drugs and cases alike where validated thresholds for resistance are not available. The underlying linear mixed model of GLSMRs allowed accounting for this variability and for unequal number of isolates collected during field testing. Based on these data GLSMRs appear to be more accurate and to offer advantages over other tests for the "longitudinal" analysis of $\mathrm{IC}_{50} \mathrm{~s}$. We used a simple statistical model which produces easily interpretable results and can be found in any statistical software. Finally, the utility of GLSMRs in monitoring drug susceptibility of not only malaria parasites but also other microbes should be further tested.

\section{Competing interests}

The author(s) declare that they have no competing interests.

\section{Authors' contributions}

All authors read and approved the final manuscript.

MV conceived the methodology and conducted the analyses; PO contributed to the concept; both contributed to the writing of the paper. PO is a staff member of the World Health Organization. The authors alone are responsible for the views expressed in this publication and they do not necessarily represent the decisions, policy or views of the World Health Organization.

\section{Acknowledgements}

The authors wish to thank Professor Philippe Brasseur for providing the data on which this work was done and Dr Pascal Millet and Professor Denis Malvy for reviewing the paper.

Data collection was part of a study (Principal Investigator: P.Brasseur) funded by the French Ministry of Foreign Affairs (FAC 2000) and Ministry of Research (PAL+), and the UNICEF/UNDP/WB/WHO Special Programme for Research \& Training in Tropical Diseases (TDR). The article processing charge of this manuscript was funded by the Fond National de la Recherche, Luxembourg.

Part of this work was presented at the 2006 Parasitology Colloquium of the French society of Parasitology in Bordeaux and at the 2006 Epidemiology Congress of the "Association des Epidémiologistes de Langue Française".

\section{References}

I. Susceptibility of Plasmodium falciparum to antimalarial drugs. Report on global monitoring 1996-2004. Geneva: World Health Organization; 2005.

2. Turnidge J, Kahlmeter G, Kronvall G: Statistical characterisation of bacterial wild-type MIC value distributions and the determination of epidemiological cut-off values. Clin Microbiol Infect 2006, I 2(5):4|8-425.

3. EUCAST Definitive Document E.DEF 2.I, August 2000 : Determination of antimicrobial susceptibility test breakpoints. Clin Microbiol Infect 2000, 6( I 0):570-572.

4. Pradines B, Tall A, Parzy D, Spiegel A, Fusai T, Hienne R, Trape JF, Doury JC: In-vitro activity of pyronaridine and amodiaquine against African isolates (Senegal) of Plasmodium falciparum in comparison with standard antimalarial agents. J Antimicrob Chemother 1998, 42(3):333-339.

5. Wongsrichanalai C, Wimonwattrawatee $T$, Sookto $P$, Laoboonchai $A$, Heppner DG, Kyle DE, Wernsdorfer WH: In vitro sensitivity of Plasmodium falciparum to artesunate in Thailand. Bull World Health Organ 1999, 77(5):392-398.

6. Brockman A, Price RN, van Vugt M, Heppner DG, Walsh D, Sookto P, Wimonwattrawatee T, Looareesuwan S, White NJ, Nosten F: Plasmodium falciparum antimalarial drug susceptibility on the north-western border of Thailand during five years of extensive use of artesunate-mefloquine. Trans $R$ Soc Trop Med Hyg 2000, 94(5):537-544.

7. Navaratnam V, Mansor SM, Mordi MN, Akbar A, Abdullah MN: Comparative pharmacokinetic study of oral and rectal formulations of artesunic acid in healthy volunteers. Eur J Clin Pharmacol | 998, 54(5):4| |-4|4.

8. Newton PN, van Vugt M, Teja-Isavadharm P, Siriyanonda D, Rasameesoroj $M$, Teerapong P, Ruangveerayuth R, Slight T, Nosten F, Suputtamongkol Y, Looareesuwan S, White NJ: Comparison of oral artesunate and dihydroartemisinin antimalarial bioavailabilities in acute falciparum malaria. Antimicrob Agents Chemother 2002, 46(4): I I25- I I27.

9. Olliaro PL, Nair NK, Sathasivam K, Mansor SM, Navaratnam V: Pharmacokinetics of artesunate after single oral administration to rats. BMC Pharmacol $2001,1: 12$.

10. Guidance for Industry: Statistical Approaches to Establishing Bioequivalence. U.S. Department of Health and Human Services, Food and Drug Administration, Center for Drug Evaluation and Research (CDER); 2001 .

II. Rani S, Pargal A: Bioequivalence: An Overview of Statistical Concepts. Indian J Pharmacol 2004, 4:209-2I6.

12. Brown H, Prescott R: Applied mixed models in Medicine - 2nd edition. Chichester: John Wiley \& Sons Ltd; 2006.

13. Antimalarial drug combination therapy. WHO; 200I.

14. Agnamey P, Brasseur P, de Pecoulas PE, Vaillant M, Olliaro P: Plasmodium falciparum in vitro susceptibility to antimalarial drugs in Casamance (southwestern Senegal) during the first 5 years of routine use of artesunate-amodiaquine. Antimicrob Agents Chemother 2006, 50(4): I53 I-I534.

15. Verbeke G, Molenberghs G: Linear mixed models for longitudinal data. New York: Springer; 2000.

16. SAS Institute Inc: The GENMOD Procedure. In SAS/STAT® 9I User's guide Volume 3 I. Cary, N.C.: SAS Institute Inc; 2004: I 62 I - I 742.

17. Brasseur P, Druilhe P, Kouamouo J, Brandicourt O, Danis M, SR M: High level of sensitivity to choloroquine of 72 Plasmodium falciparum isolates from southern Cameroon in January 1985. Am J Trop Med Hyg 1986, 35:71 I-716.

18. Agnamey P, Brasseur P, Cissé M, Gaye O, Dumoulin J, Rigal J, Taylor WRJ, Olliaro P: Economic evaluation of a policy change from single-agent treatment for suspected malaria to artesunateamodiaquine for microscopically confirmed uncomplicated falciparum malaria in the Oussouye district of southwestern Senegal. Trop Med Int Health 2005.

19. Desjardins RE, Canfield CJ, Haynes JD, Chulay JD: Quantitative assessment of antimalarial activity in vitro by a semiautomated microdilution technique. Antimicrob Agents Chemother 1979, 16:710-718.

20. SAS Institute Inc: The MIXED Procedure. In SAS/STAT® $9 /$ User's guide Volume 31. Cary, N.C.: SAS Institute Inc; 2004:267I-2863.

21. Kaddouri H, Nakache S, Houze S, Mentre F, Le Bras J: Assessment of the drug susceptibility of Plasmodium falciparum clinical isolates from africa by using a Plasmodium lactate dehydroge- 
nase immunodetection assay and an inhibitory maximum effect model for precise measurement of the 50 -percent inhibitory concentration. Chemother 2006, 50(10):3343-3349.

22. Stepniewska K, Chotivanich K, Brockman A, Day NP, White NJ: Overestimating resistance in field testing of malaria parasites: simple methods for estimating high EC50 values using a Bayesian approach. Malar J 2007, 6:4.

23. Box GEP, Cox DR: An Analysis of Transformations. Journal of the Royal Statistical Society Series B (Methodological) 1964, 26(2):2 I I-252.

Publish with Biomed Central and every scientist can read your work free of charge

"BioMed Central will be the most significant development for disseminating the results of biomedical research in our lifetime. " Sir Paul Nurse, Cancer Research UK

Your research papers will be:

- available free of charge to the entire biomedical community

- peer reviewed and published immediately upon acceptance

- cited in PubMed and archived on PubMed Central

- yours - you keep the copyright

Submit your manuscript here:

http://www.biomedcentral.com/info/publishing_adv.asp
BioMedcentral 